\title{
Papillary Carcinoma Derived from Ovarian Mature Cystic Teratoma: A New Case Report and Literature Review
}

\author{
Ihsane Souaf, Hinde El Fatemi, Amal Bennani, Chbani Leila, Hammas Nawale, \\ Harmouch Tawfik, Afaf Amarti \\ Department of Pathology, Hassan II Teaching Hospital, Fez, Morocco \\ Email: souaf.ihsane@gmail.com
}

Received 16 February 2014; revised 10 March 2014; accepted 1 April 2014

Copyright (C) 2014 by authors and Scientific Research Publishing Inc.

This work is licensed under the Creative Commons Attribution International License (CC BY).

http://creativecommons.org/licenses/by/4.0/

c) (i) Open Access

\begin{abstract}
Introduction: Papillary carcinoma arising from thyroid tissue in mature cystic teratoma (MCT) of the ovary is extremely rare, and it is not easy to diagnose preoperatively. No clinical, no radiological, no biological signs are specific to malignant transformation. Case presentation: A 51-yearold woman had a left ovarian tumor measuring approximately $18 \mathrm{~cm}$ diameter with malignant transformation of a mature cystic teratoma, who was diagnosed postoperatively with follicular variant of papillary thyroid carcinoma. A laparoscopic hysterectomy and left salpingo-oophorectomy were performed. During surgery, there were no signs of invasion or metastasis. Conclusion: Consideration should be given to thyroidectomy followed by total-body scanning and serum studies for foci of thyroid carcinoma and adjuvant therapy with thyroidectomy and radio ablation if residual disease is identified.
\end{abstract}

\section{Keywords}

Ovarian Teratoma, Papillary Carcinoma, Malignant Transformation, Thyroid, Ovarian Neoplasm

\section{Introduction}

Mature cystic teratoma commonly referred to as a dermoid cyst of the ovary. It is the most common type of ovarian teratoma and germ cell neoplasm comprising $10 \%-20 \%$ of ovarian tumors in the female reproductive period [1]. Usually unilateral, it is, by definition, composed of well-differentiated derivates which originate from all three layers. It is most frequently diagnosed, in its pure form, it is generally benign, but malignant transfor- 
mation has been reported, rarely, in one of its elements with a rate $1 \%$ - $2 \%$ [2], always in postmenopausal women, however, it can also be rarely identified in younger women [1].

The most common malignant tumor in mature cystic teratomas is squamous cell carcinoma, whereas, papillary thyroidcarcinoma is extremely rare. Malignant transformation is rarely diagnosed preoperatively, due to its rarity [3], only by postoperative pathological examination. In this paper, we report a rare case of follicular variant papillary thyroid cancer arising from a mature cystic teratoma of the ovary, which was unexpectedly discovered during histopathologic examination.

\section{Case Report}

A 51-year-old woman, with no significant medical history, was admitted in gynecology with a pelvic pain associated with signs of digestive compression occurring in a context of poorgeneral condition.

Her physical examination revealed a abdominal pelvic mass beyond the umbilicus, hard consistency, movable relative to both superficial and deep planes.

Ultrasound of the abdomen revealed a large multilobular mixed cystic and solid abdomino-pelvic mass $18 \times$ $15 \mathrm{~cm}$, containing hyperechogenic areas, calcifications were noted, densely adherent to the intestinal and uterine wall with moderateascitis.

The intraoperative exploration found a large tumor appeared to have originated from the left ovary and adherent to hail. A hysterectomy and left salpingo-oophorectomy with resection of the small intestine were performed.

Macroscopic appearance of the lesion resembled a dermoid cyst, color yellowish, seat alterations and necrotic hemorrhagic and contained cystic microcavities colloid content.

Histopathologic evaluation of the lesion demonstrated a follicular variant papillary thyroidcarcinoma in a mature cystic teratoma and surrounded by normal ovarian tissue with a intact capsule.

Microscopic examination revealed a mature cystic teratoma with multiple focus of follicular variant papillary thyroid cancer (Figure 1 and Figure 2) surrounded by normal ovarian tissue. Sections of normal thyroid parenchyma, borne, cartilage and respiratory mucosa were identified in this own proliferation (Figure 3). There was no immature tissue inside the teratoma. Immunohistochemistry demonstrated, tumor cells expressing the TTF1, thyroglobulin (Figure 4) and cytokeratin 19 (Figure 5).

The diagnosis was of Follicular variant of papillary thyroid carcinoma arising from a Mature cystic teratoma.

Thyroid ultrasonography was necessary to eliminate any primary thyroid malignancy and serum thyroid function tests were normal.

Tyroidectomy was performed and pathological examination of the thyroid gland was normal. The patient was

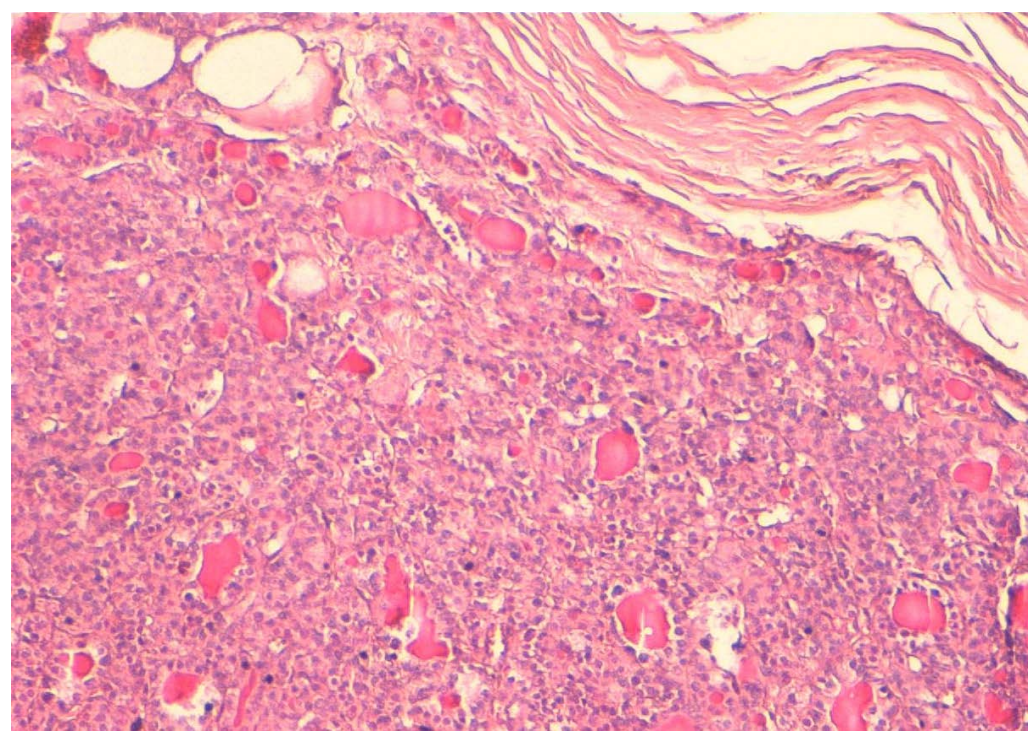

Figure 1. Tumor proliferation arranged in sheets and trabecular with drafts of vesicular structures although limited by a fibrous capsule $(\mathrm{HE} \times 10)$. 


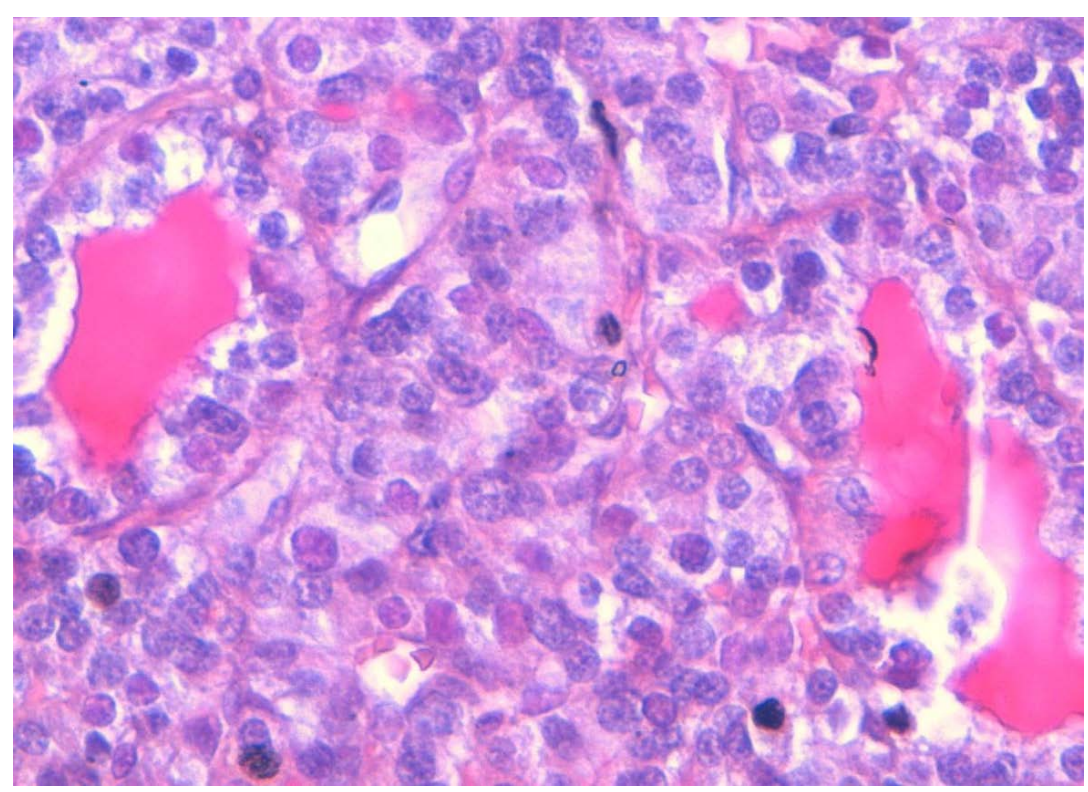

Figure 2. Tumor cells are ovoids, moderately atypicals. The nuclei increased in size, clarified and overlapping $(\mathrm{HE} \times 20)$.

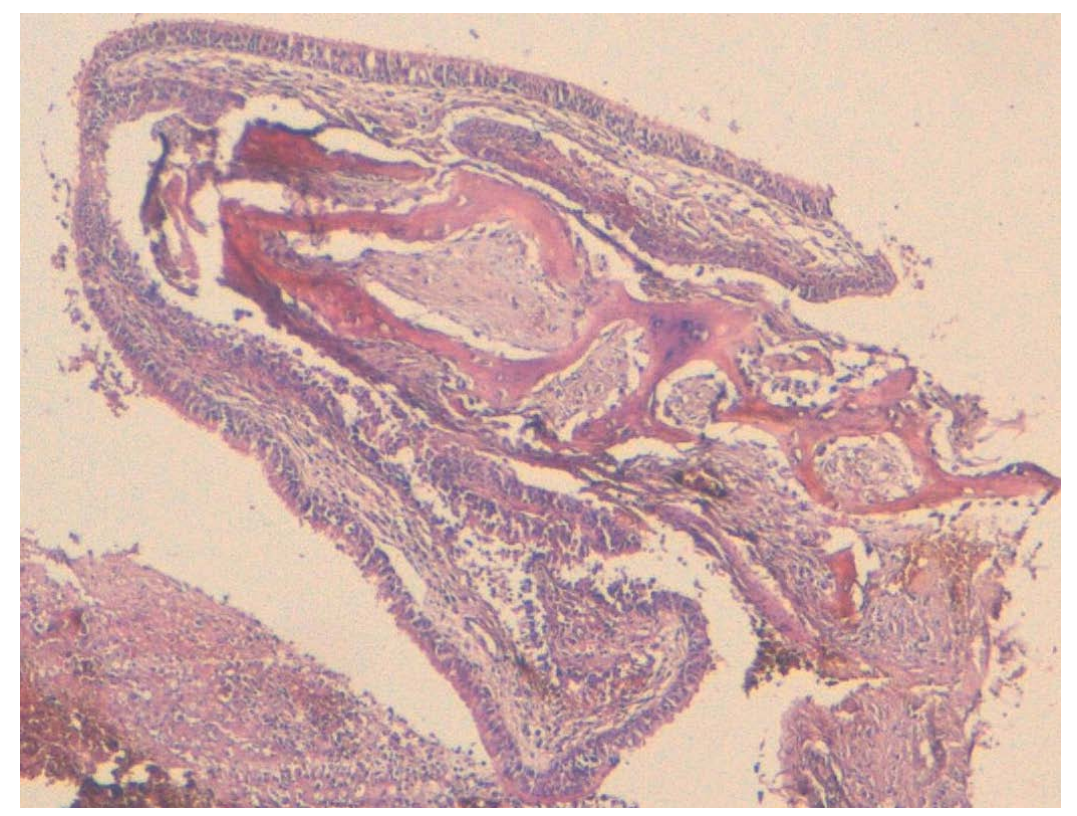

Figure 3. Section of borne, cartilage and respiratory mucosa $(\mathrm{HE} \times 10)$.

under surveillance for 6 months, with no evidence of recurrence.

\section{Discussion}

Mature cystic teratoma of the ovary is the most common germ cell tumour accounting for a third of all ovarian tumours and ninety-five per cent of these cases are benign. Usually unilateral, it is by definition composed of well-differentiated derivates (like teeth, hair, bone and thyroid tissue) which originate from all three germ cell layers and is most frequently diagnosed during the female reproductive period [4]. Complications of cysticteratomas include torsion (16\%), malignant degeneration (2\%), rupture (1\% - 2\%), and infection (1\%) [5].

A malignant transformation of the mature cystic teratoma is an uncommon complication and occurs in $1 \%$ - 


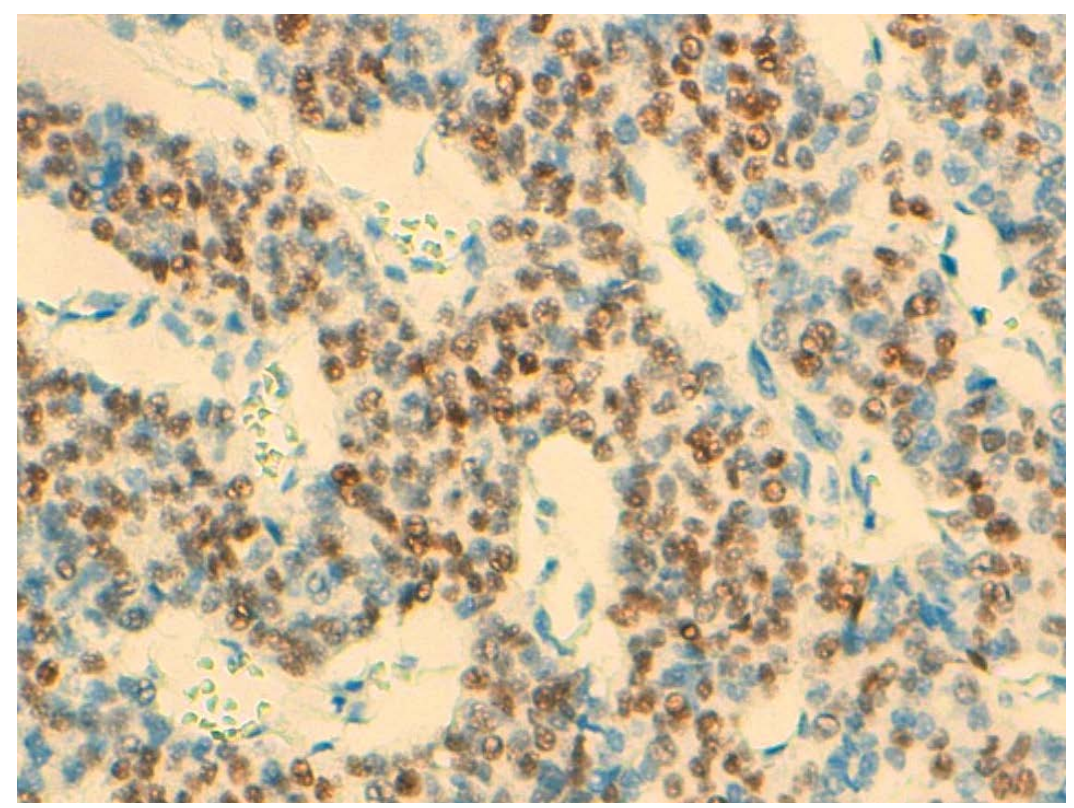

Figure 4. Tumor cells expressed TTF1 $(\mathrm{HE} \times 10)$.

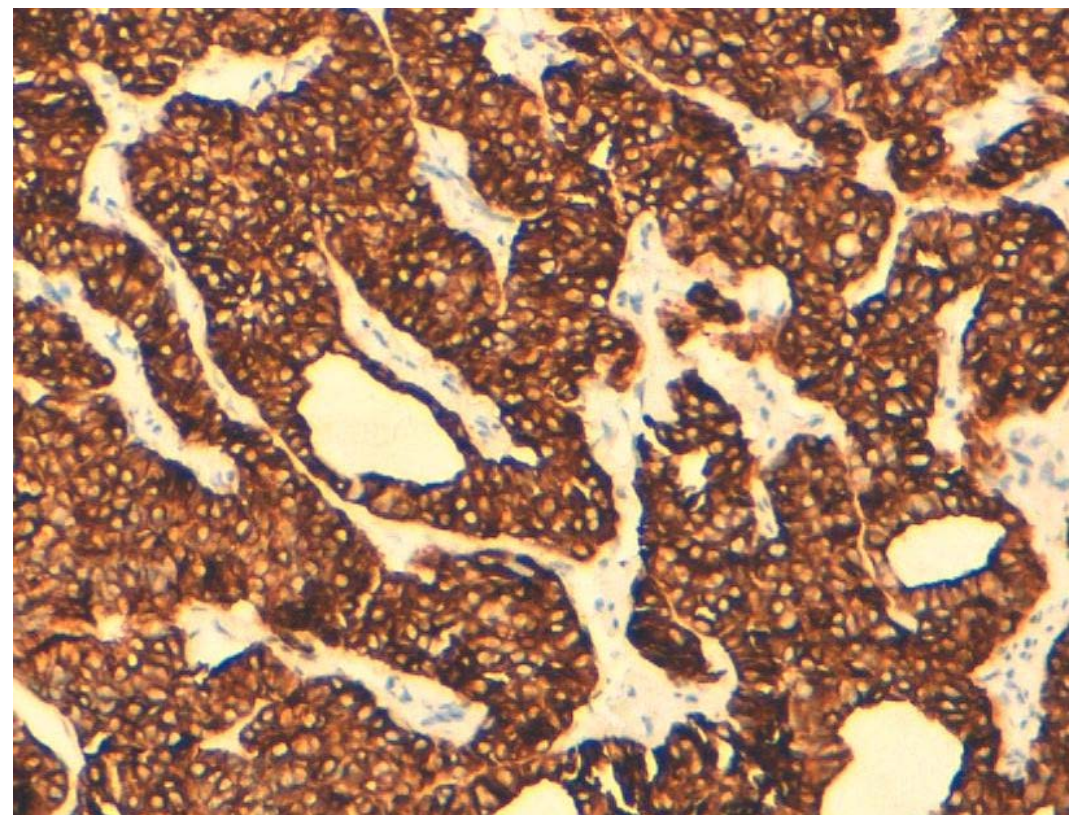

Figure 5. The tumor cells expressed cytokeratin $19(\mathrm{HE} \times 10)$.

$2 \%$ of cases, most frequently in postmenopausal women [6]. Usually diagnosed postoperatively, from the histological findings [1]. Though malignant transformation can occur from any of the embryonic germ layers, the most common malignancy arising in these otherwise benign tumours is squamous cell carcinoma [7].

In 1957, Peterson reviewed 229 cases of malignant transformation of mature cystic teratomas [8]. He reported malignant transformation rates of $1.8 \%$; $81.1 \%$ of the tumors were squamous cell carcinomas and $6 \%$ were adenocarcinomas. The other malignant transformations include malignant melanoma, sarcoma, basal cell carcinoma, carcinoid tumor, adenocarcinoma of intestinal epithelium, and thyroid carcinoma [1].

The presence of thyroid tissue in the ovaries was first described by Von Kahlden in 1895 [9]. Papillary thyroid cancer arising from ovarian teratomas is a rare occurrence, with an estimate of $0.1 \%-0.3 \%$ [10]. To date, only 15 cases of papillary thyroid carcinoma arising in a mature cystic teratoma of the ovary have been reported. 
The average age was 45 years. These patients predominantly presented with abdominal pain (50\%), a pelvic mass (12\%) and menstrual irregularities (12\%). Papillary carcinoma was the most common (60\%) histopathological finding followed by follicular variant of papillary carcinoma (33\%) and follicular carcinoma (6\%) [1]. Most patients present a pelvic mass, and pelvic ultrasonography characteristically shows a heterogeneous, solid mass, occasionally with ascites.

Malignant transformation of thyroid tissue in mature cystic teratoma can be classified Histopathologically into three types [11]. The first and most common type is papillary carcinoma (44\%). The malignant features of these tumors include optically clear or ground-glass overlapping nuclei with nuclear grooves. The second type is follicular carcinoma, which is diagnosed when cells containing mitotic figures begin forming follicles and capsular or vascular invasion is noted. Follicular carcinoma accounts for approximately $30 \%$ of malignant degeneration in MCT. Lastly, there is a follicular variant of a papillary carcinoma, which accounts for $26 \%$ of malignant degeneration. This follicular variant is unique because its hares similar nuclear cytological features of a papillary carcinoma; it has, however, a follicular architecture.

In our case, a follicular variant papillary thyroid carcinoma was found. It is a teratoma with a thyroid tissue, where we found a area with typical histological characteristics of a follicular variant papillary thyroidcarcinoma and positive TTF1, cytokeratine 19, thyroglobulin on immunohistochemical analysis.

The survival outcome of patients with malignant transformation of the ovarian mature cystic teratomas is dependent on the stage. These tumors arising from ovarian teratomas can occasionally present with locally invasive disease, or with distant metastases, and may be associated with recurrences in some patients. In the present case, a lesion was found on the left ovary, with ascite and adherent to the intestinal without rupture or tumor dissemination.

Conservative treatment is often offered to younger patients who wished to retain their fertility and it consists of unilateral salpingo-oophorectomy or cystectomy without adjuvant therapy. Conservation of ovarian tissue on the affected side is acceptable [1].

Thyroidectomy was necessary not only to confirm normal thyroid, but also to facilitate nuclear medicine imaging, so that radioactive iodine uptake would occur mainly in metastatic thyroid tissue. Later this would also have potentiated therapy if metastases were detected [12].

Serial measurement of serum thyroglobulinis a sensitive and it should be used for long-term follow-up, up to 10 years to detect metastasis or tumor recurrence [13]. Serumthy roglobulin levels are checked every 6 months during the 1st year after surgery. If a patient's thyroglobulin level is unexpectedly high, a radio-active iodine scan is carried out, in order to evaluate for recurrence [1].

The optimal treatment for malignant transformation of mature cystic teratoma is uncertain because of the rarity of the disease [14]. This rarity suggests a low likelihood of future prospective studies to determine the best treatment options.

Early detectionis important for long-term survival. Old age, large tumor size, and solid portion in mature cystic teratoma seem to predict the malignant transformation of mature cystic teratoma [15].

\section{Conclucion}

Due to the paucity of cases published, it is difficult to predict the natural progression of this disease, its management and the long-term prognosis.

\section{References}

[1] Dane, C., Ekmez, M., Karaca, A., et al. (2012) Follicular Variant of Papillary Thyroid Carcinoma Arising from a Dermoidcyst: A Rare Malignancy in Young Women and Review of the Literature. Taiwanese Journal of Obstetrics and Gynecology, 51, 421-425. http://dx.doi.org/10.1016/j.tjog.2012.07.019

[2] Wen, K.C., Hu, W.M., Twu, N.F., et al. (2006) Poor Prognosis of Intraoperative Rupture of Mature Cystic Teratoma with Malignant Transformation. Taiwanese Journal of Obstetrics and Gynecology, 45, 253-256. http://dx.doi.org/10.1016/S1028-4559(09)60236-9

[3] Mori, Y., Nishii, H., Takabe, K., et al. (2003) Preoperative Diagnosis of Malignant Transformation Arising from Mature Cysticteratoma of the Ovary. Gynecologic Oncolgy, 90, 338-341.

[4] Prasad, P., Nunns, D., Ubhi, C.S., et al. (2008) A Rare Case of Follicullar Thyroide Carcinoma in Ovarian Stroma. The Royal Colleges of Surgeons of Edinburgh and Ireland, 5, 313-315. 
[5] Lipson, S.A. and Hricak, H.M. (1996) Imaging of the Female Pelvis. Radiologic Clinics of North America, 34, 1157-1182.

[6] Christopherson, W.A. and Councell, R.B. (1989) Malignant Degeneration of a Mature Ovarian Teratoma. International Journal of Gynecology \& Obstetrics, 30, 379-384.

[7] Levine, D.A., Villela, J.A., Poynor, E.A., et al. (2004) Gastro Intestinal Adenocarcinoma Arising in a Mature Cysticteratoma of Ovary. Gynecologic Oncology, 94, 597-593. http://dx.doi.org/10.1016/j.ygyno.2004.05.045

[8] Peterson, W.F. (1957) Malignant Degradation of Benign Cystic Teratomas of the Ovaryda Collective Review of the Literature. Obstetrical \& Gynecological Survey, 12, 793-830. http://dx.doi.org/10.1097/00006254-195712000-00001

[9] Von Kahlden, C. (1895) Ueber Eine Eigenthumliche Form des Ovarialcarcinoms. Zentralblallg Path, 7, 257.

[10] Rim, S.Y., Kim, S.M. and Choi, H.S. (2006) Malignant Transformation of Ovarian Mature Cystic Teratoma. International Journal of Gynecological Cancer, 16, 140-144. http://dx.doi.org/10.1111/j.1525-1438.2006.00285.X

[11] Kurman, R. and Telinde, R. (2002) Blaustein’s Pathology of the Female Genital Tract. 5th Edition, Springer Verlag, New York.

[12] Navarro, M.D., Tan, M.L.A., Lovecchio, J.L., et al. (2004) Malignant Struma Ovarii: Case Report. Annals of Clinical \& Laboratory Science, 34, 107-111.

[13] Anita, A., Jill, N., Maire, A., et al. (2010) Papillary Thyroid Carcinoma Arising in a Struma Ovarii: A Case Report and Analysis of Cases Reported in the Literature. Clinical Ovarian Cancer, 3, E6-E10.

[14] Al-Nawafleh, A. and Khreisat, A.H. (2010) Papillary Thyroid Carcinoma Arising in a Mature Cysticteratoma. Journal of the Royal Medical Service, 17, 91-93.

[15] Jeong-Yeol, P., Dae-Yeon, K., Jong-Hyeok, K., et al. (2008) Malignant Transformation of Mature Cysticteratoma of the Ovary: Experienceat a Single Institution. European Journal of Obstetrics \& Gynecology and Reproductive Biology, 141, 173-178. http://dx.doi.org/10.1016/j.ejogrb.2008.07.032 